\title{
Lecture demonstrations of interference and quantum erasing with single photons
}

\author{
T L Dimitrova ${ }^{1,2}$ and A Weis ${ }^{2}$ \\ ${ }^{1}$ University of Plovdiv "Paissi Hilendarski", Tzar Assen Street 24, BG-4000 Plovdiv, Bulgaria \\ ${ }^{2}$ Physics Department, University of Fribourg, Chemin du Musée 3, CH-1700 Fribourg, Switzerland \\ E-mail: doradimitrova@uni-plovdiv.bg and antoine.weis@unifr.ch
}

\begin{abstract}
Single-photon interference is a beautiful manifestation of the wave-particle duality of light and the double-slit Gedankenexperiment is a standard lecture example for introducing quantum mechanical reality. Interference arises only if each photon can follow several (classical) paths from the source to the detector, and if one does not have the possibility to determine which specific path the photon has taken. Attaching a specific label to the photon traveling along a specific path destroys the interference. However, in some cases those labels can be erased from the photon between leaving the apparatus and being detected, by which interference can be restored, a phenomenon called quantum erasing. We present lecture demonstration experiments that illustrate the wave-particle duality of light and the phenomenon of quantum erasing. Both experiments are first shown with strong light and, in a second step, on a photon-by-photon basis. The smooth transition from the quantum to the classical case can be shown in real time by varying the incident light intensity.
\end{abstract}

PACS numbers: 42.50.-p, 03.67.Bg, 03.65.Ta, 42.50.Xa, 01.40.Fk, 01.40.gb

\section{Introduction}

Wave-particle duality is a main feature of quantum mechanical concepts, offering a unified picture of seemingly contradictory classical representations of reality. Its most convincing manifestation is the phenomenon of single-photon interference. In recent years, the authors have developed two lecture demonstration experiments of single-photon interference: double-slit interference with detection by a single-photon CCD camera [1,3] and two-path interference in a Mach-Zehnder interferometer (MZI) with photomultiplier detection $[2,3]$. Both demonstrations show the simultaneous existence of wave properties and of particle properties of light. This illustrates that those complementary properties do not depend on the nature of the performed experiment, but are inherent properties of a quantum system.

In addition, the outcome of a quantum mechanical experiment may depend on how and when something is measured, and there is an inextricable link between information (the knowledge we have) of the investigated system and its real physical state. In the words of Paul
Kwiat [4]: 'Quantum information, in the end, describes not only what can be known, but the subtle effect that knowing has on nature.' The 'classical' experiment that addresses this issue is the quantum eraser, proposed by Scully and Drühl [5] in 1982, and further refined in different realizations [6].

The experiments presented here are based on a two-path MZI, which is well suited to demonstrating quantum interference [2, 3, 7] and single-photon quantum erasing. In the most widely accepted Copenhagen interpretation of quantum mechanics, the photon in the apparatus is described by a delocalized probability wave that evolves along the two classical trajectories. Interference arises as a consequence of the phase difference accumulated by the two coherent partial waves. The wavefunction description allows one to make only probabilistic statements of the photon's state inside of the apparatus, whereas in the de Broglie-Bohm picture [8] the photon takes a well-defined path while being guided by a quantum mechanical pilot wave that follows both paths, thereby determining the interference. It is well known that any experimental feature (labeling mechanism) that would allow the determination of the photon path 

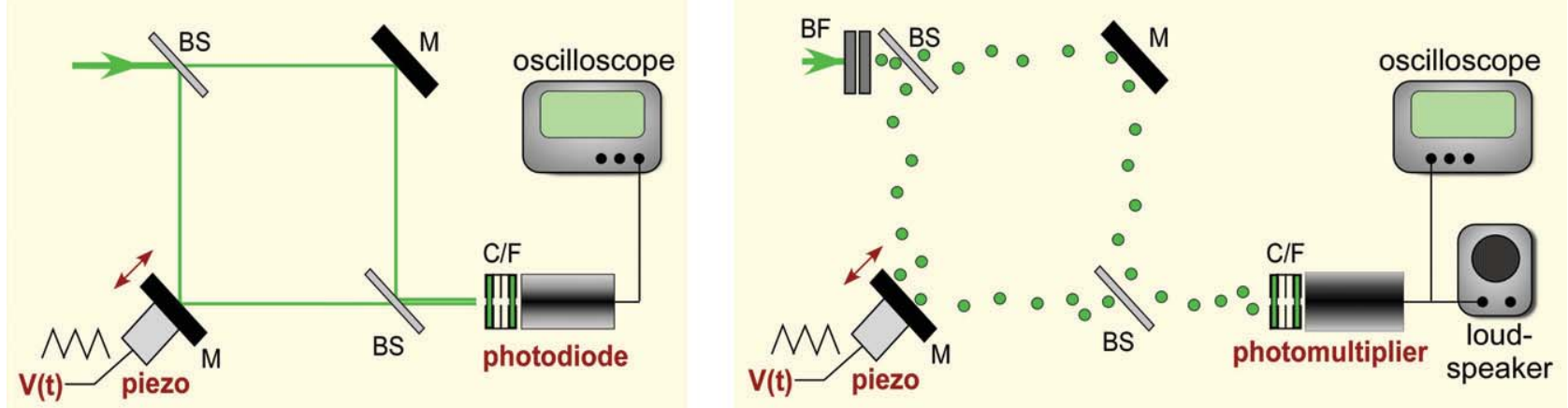

Figure 1. Experimental setup. Left: classical interference with strong laser light in scanning mode with photodiode detection (M: mirror; BS: beam splitter; C/F: collimator-filter box). Right: single-photon interference with photomultiplier detection (BF: attenuating filter).

inevitably destroys the coherence of the probability wave, and hence interference. In some cases, however, it is possible to erase that which-way information after the photon has left the apparatus, thereby restoring interference, a phenomenon called quantum erasing.

A simple way of marking the possible paths in the MZI is the insertion of orthogonally oriented linear polarizers in the two interferometer arms. As a consequence the interference pattern disappears. In practice, the erasing of the which-way information is realized by inserting before the (detecting) screen a third polarizer oriented at $\pm 45^{\circ}$, called the 'eraser'. This erasing polarizer destroys the which-way information imposed by polarization labels and makes the interference reappear, a phenomenon that, when observed on a photon-by-photon basis, is a manifestation of quantum erasing. In terms of classical wave superposition the phenomenon is readily understood, but its understanding at the single-photon level presents some difficulties for students. From a didactical point of view, it is a nice example for introducing the concept of entanglement: while being in the interferometer the external degree of freedom (path) of each photon's wavefunction is entangled with its internal degree of freedom (polarization). Although quantum erasing is even more puzzling in a version using entangled photon pairs, we believe that single-photon quantum erasing is most suited for a lecture introduction of the phenomenon.

Recently, we have extended our lecture demonstration experiment on the wave-particle duality of light for demonstrating the phenomenon of quantum erasing with single photons.

\section{Single-photon interference}

In our previous work [3] we have presented lecture demonstration experiments that show the wave-particle duality by comparing classical (strong light) interference with quantum (single-photon) interference. The motivation for the elaboration of this experiment was to show in a single experiment the wave and the particle properties of light and to demonstrate the coexistence of these two contradictory aspects of light. We now use a simplified version (shown in figure 1) of the apparatus described in [3] that has only a single beam traversing an MZI. The light source is a $5 \mathrm{~mW}$ green laser module with a wavelength close to the maximum of the eye's spectral sensitivity. As discussed in [3, 7] the use of an MZI rather than a double-slit presents some methodological and didactical advantages for demonstrating quantum interference. Its spatially well-separated beams facilitate light manipulations in the two paths.

Conventionally, the wave nature of light is presented by projecting the interference pattern on a screen (projection mode). Alternatively, one may displace one of the MZI mirrors by a piezotransducer (figure 1 , left) controlled by a periodic linear voltage ramp (scanning mode). In this way the difference between the optical paths of the two arms of the interferometer undergoes a periodic linear change whose effect can be detected as a modulation of the light intensity recorded by a photodiode with a small entrance aperture. The interference pattern is represented via the sinusoidal variation of the photocurrent displayed by an oscilloscope.

For the single-photon experiments (figure 1, right), we insert strongly attenuating filters as well as a filter of variable transmission before the entrance of MZI. A single-photon detecting photomultiplier equipped with a collimator and filters that strongly suppress stray light allow the demonstration to be performed in an ambient light environment. The photomultiplier pulses are sent to an oscilloscope and to a loudspeaker so that single-photon events can be both seen and heard.

Strong light interference is first shown as oscilloscope fringes from the photodiode in the scanning mode (lower trace of figure 2). After insertion of the attenuating filters, single-photon interference is shown in the same way using the photomultiplier signal. The top three traces of figure 2 show the photomultiplier signals in a single sweep of the piezo and after multiple averages of subsequent sweeps.

In the top trace individual photons are seen as pulses on an oscilloscope time trace. While the individual pulses represent particle nature, their (temporal) density is a measure of light intensity. When the number of averaged traces is increased, individual pulses pile up and a periodic modulation of the pulse density appears. This modulation becomes smoother and smoother and approaches the photodiode signal asymptotically as more and more traces are added. In this way one sees how classical wave interference emerges gradually from single-particle interference events, convincing evidence for the wave-particle duality of light.

A most spectacular demonstration consists in adjusting the interferometer (in photon projection mode) such that 


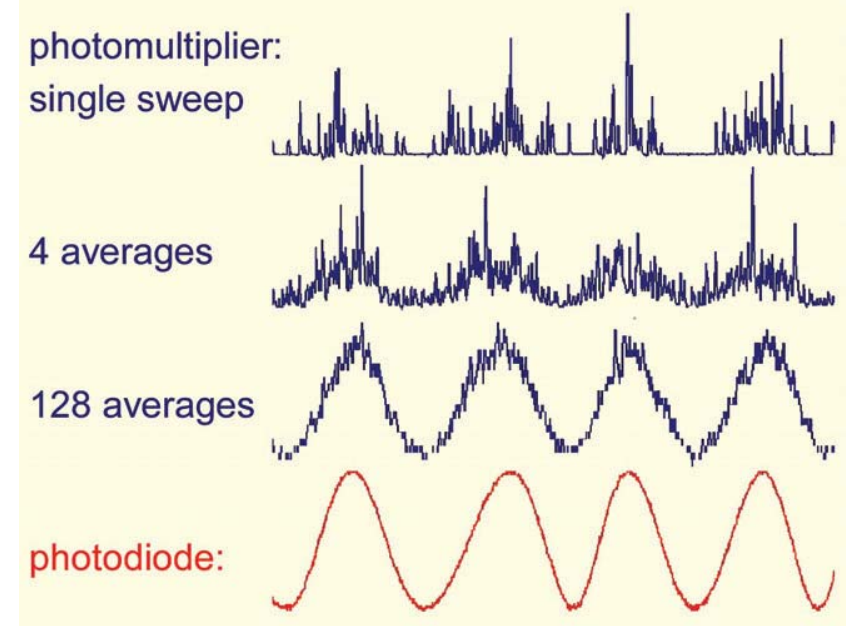

Figure 2. From single-photon interference (top) to wave interference (bottom). The classical interference pattern emerges when many single-photon traces are averaged.

the photomultiplier is located in an interference minimum characterized by a very low click rate of the loudspeaker signal. When one of the paths of the MZI is then blocked by insertion of a large cardboard, the click rate increases in a dramatic way, thereby demonstrating that indeed each photon interferes with itself (according to the Copenhagen interpretation).

\section{Quantum erasing}

For labeling of the which-path information required in the demonstration of quantum erasing, orthogonal linear polarizers $\mathrm{P} 1$ and $\mathrm{P} 2$ are inserted in the two paths as shown in figure 3 . The experiment would be ideally performed with unpolarized light. However, since laser light is always strongly (albeit not perfectly) polarized we insert a prepolarizer oriented at $\pm 45^{\circ}$ with respect to the horizontal. The erasing polarizer P3, mounted on a rotation stage, is inserted at one of the exits of MZI. This arrangement allows the demonstration of quantum erasing both with classical waves (strong light) and with single photons in a strongly attenuated beam. We use both exits of the interferometer. The erasing of the labels is performed on one of them, while the labeling effect remains visible on the second exit throughout the experiment (figure 3).

We begin the demonstration by showing quantum erasing with strong light. The path labeling by the polarizer destroys the interference, which is well seen by screen projection. After insertion of the eraser oriented at $+45^{\circ}$ or $-45^{\circ}$ the interference pattern reappears with full contrast (figure 4, top), although its overall intensity is reduced by a factor of 2 . By rotating the erasing polarizer away from the $\pm 45^{\circ}$ orientation, the contrast is reduced and disappears completely at $0^{\circ}$ (figure 4 , top) and $90^{\circ}$, in which case only light from one of the paths reaches the detector. This wave erasing phenomenon can be easily explained by classical wave mechanics which yields for the intensity on the screen

$$
I=\frac{I_{0}}{4}(1-\cos \Delta \phi \sin 2 \alpha),
$$
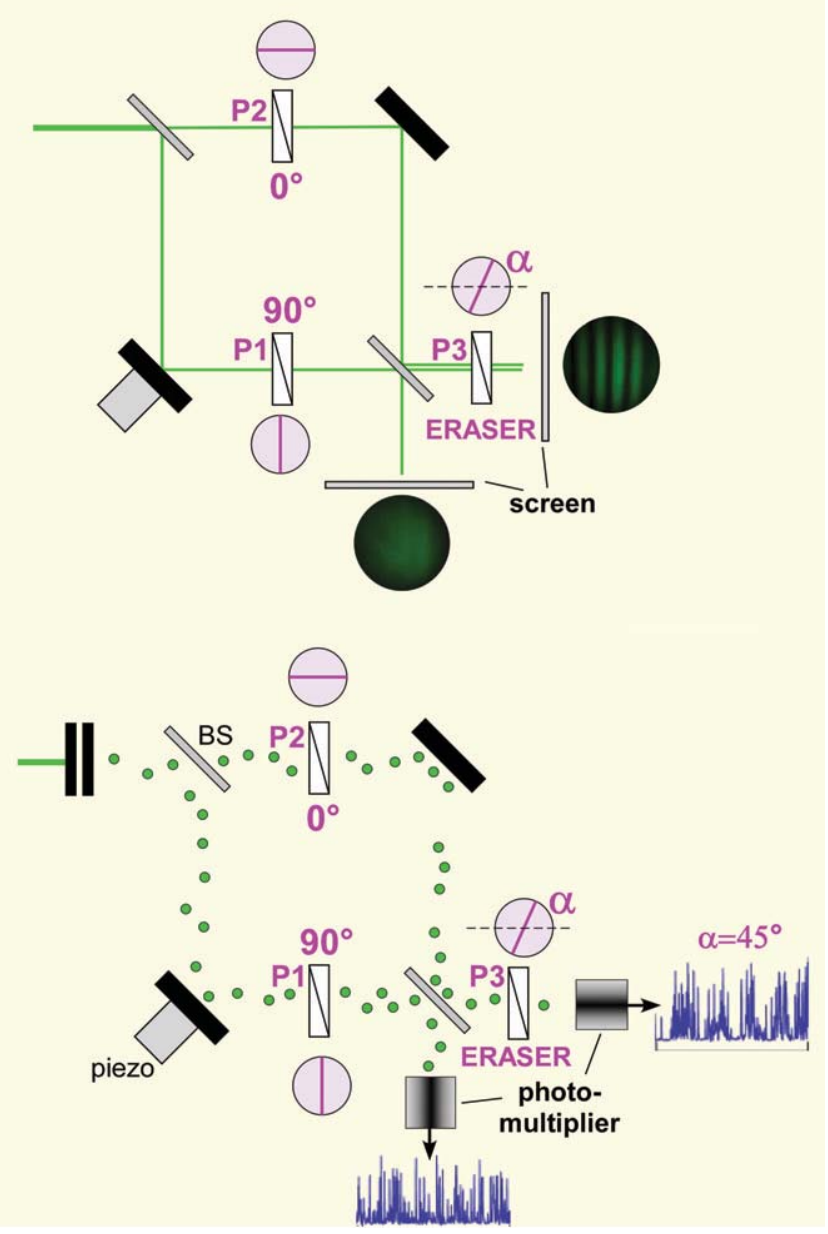

Figure 3. Top: wave erasing with strong light. Bottom: quantum erasing with single photons.

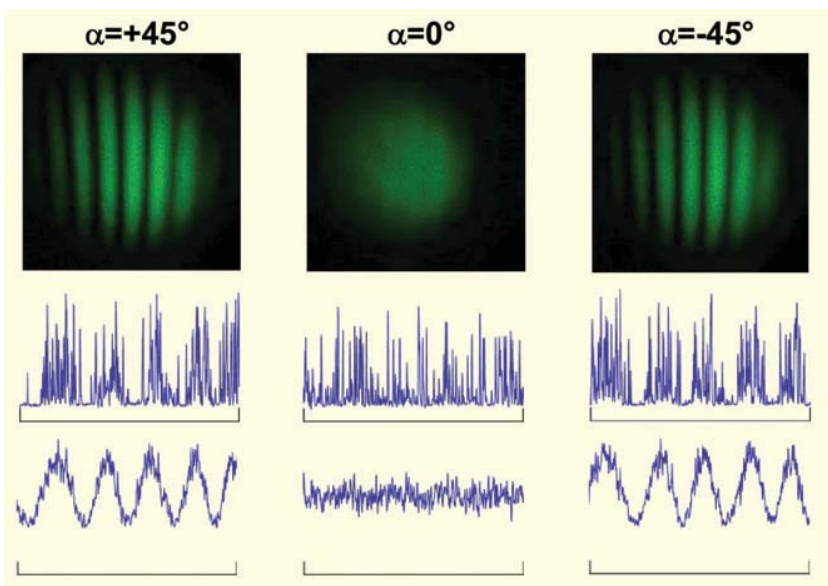

Figure 4. Top line: wave erasing in screen projection mode. Middle line: quantum erasing with photons, recorded as a single oscilloscope trace of the photomultiplier pulses. Bottom line: averaged oscilloscope traces.

where $\alpha$ is the angle of the eraser's axis with respect to the horizontal and $\phi$ is the phase difference of the interfering beams, which is proportional to the coordinate of the fringe position on the screen.

Wave erasing can also be shown in scanning mode. Without labeling polarizers in the MZI, one sees a sinusoidal 
fringe pattern on the oscilloscope. When the prealigned polarizers $\mathrm{P} 1$ and $\mathrm{P} 2$ are inserted, the interference disappears and the oscilloscope displays a flat trace without structure. After inserting the erasing polarizer, one sees the restored interference pattern for the $\pm 45^{\circ}$ orientations, and one can show the change of contrast when the eraser is rotated away from those orientations.

The demonstration of quantum erasing on the photonby-photon basis is done in the scanning mode with strongly attenuated light, in the same way as in the wave-particle demonstration described above.

After having shown that single-photon interference with no inserted polarizers yields a sinusoidally modulated pulse density (as in figure 2), one readily shows that the interference is made to disappear by insertion of the orthogonal polarizers $\mathrm{P} 1$ and $\mathrm{P} 2$, as is evidenced by a uniform density distribution of photon pulses. Like in the classical case, the insertion of the erasing polarizer oriented at $\pm 45^{\circ}$ makes the modulation (interference) reappear with decreasing contrast as the eraser orientation is rotated away from $\pm 45^{\circ}$ (figure 4 , middle line).

In all demonstrations one first shows a single trace and then subsequently averaged traces (figure 4, bottom line) to make the contrast of the interference structure apparent. The disappearance of the contrast in the extreme cases of $0^{\circ}$ and $90^{\circ}$ orientation of the eraser is trivial in the sense that only light from one of the labeled paths reaches the detector in those configurations, which yields a (trivial) full knowledge of the which-way information. The increase of the contrast observed from rotating the eraser away from $0^{\circ}$ or $90^{\circ}$ can be interpreted as the gradual disappearance of the which-way information.

\section{Summary}

We have presented two novel lecture demonstration experiments for the wave-particle duality of light and for the phenomenon of quantum erasing. The experimental setup allows demonstrations both with strong laser light (by screen projection or by photodiode detection) and on a photon-by-photon basis (photomultiplier detection). The wave-particle duality is shown on the basis of single-photon interference in an MZI. The which-way information in the quantum erasing experiment is applied by orthogonal linear polarizers in the two arms of the interferometer. The erasing tool is a rotatable linear polarized, placed after the exit of the interferometer.

The experiments are well suited to accompanying introductory lectures in quantum mechanics for which demonstration experiments are generally scarce. By their impressive visualizations these demonstrations help the students develop a better feeling for specific features of quantum mechanics, such as wave-particle duality, superposition and delocalization of quantum states, which-way measurements, entanglement and quantum information.

\section{Acknowledgments}

One of us (TLD) acknowledges financial support by the Fondation du Fonds de la Recherche of the University of Fribourg. We acknowledge skillful help from the mechanical and electronics workshops of the Physics Department at the University of Fribourg.

\section{References}

[1] Weis A and Wynands R 2003 Three demonstration experiments on the wave and particle nature of light PhyDid 1/2 67

[2] Dimitrova T L and Weis A 2007 A double demonstration experiment for the dual nature of light Proc. SPIE 6604 66040-O1-O5

[3] Dimitrova T L and Weis A 2008 The wave-particle duality of light: a demonstration experiment Am. J. Phys. 76137

[4] Hillmer R and Kwiat P 2007 A do-it-yourself quantum eraser Sci. Am. $\mathbf{9 0} 296$

[5] Scully M O and Drühl K 1982 Quantum eraser: a proposed photon correlation experiment concerning observation and delayed choice in quantum mechanics Phys. Rev. A 25 2208-13

[6] Walborn S P, Terra Cunha M O, Pádua S and Monken C H 2002 Double-slit quantum eraser Phys. Rev. A 65033818

[7] Scarani V and Suarez A 1998 Introducing quantum mechanics: one particle interference Am. J. Phys. 66718

[8] Bohm D and Hiley B J 1993 The Undivided Universe: An Ontological Interpretation of Quantum Theory (London: Routledge) 